\title{
TI.60.1
}

\section{IDtrust 2011 - 10th Symposium on Identity and Trust on the Internet}

- PDF: 10thAnnualIDTrustWorkshop.pdf

- Text: 10thAnnuallDTrustWorkshop.txt

\section{More Information}

\begin{tabular}{|l|l|}
\hline Repository ID & TI.60.1 \\
\hline Persistent URL & http://doi.org/10.26869/TI.60.1 \\
\hline Title & IDtrust 2011 - 10th Symposium on Identity and Trust on the Internet \\
\hline Authors & Ken Klingenstein, et al \\
\hline Sponsor & NIH, NIST, Internet2 \\
\hline Review & \\
\hline Status & Legacy \\
\hline Publish Date & April 6-7, 2011 \\
\hline DOI & $10.26869 / T I .60 .1$ \\
\hline Signature & \\
\hline Deprecated & No \\
\hline Future Review & \\
\hline Supersedes & \\
\hline Format & PDF, Text \\
\hline Related Docs & \\
\hline Development Location & \\
\hline IP Framework & \\
\hline Subject Tags & \\
\hline Notes & \\
\hline
\end{tabular}

